\title{
A practice model for provision of sexuality nursing care to gynaecological cancer patients
}

Ka-ming CHOW'1, Carmen W. H., CHAN1, Kai-chow CHOI', Isabel D WHITE², Ka-yi, SIU³, Wai-ha SIN4

${ }^{1}$ The Nethersole School of Nursing, The Chinese University of Hong Kong, Hong Kong SAR, China

${ }^{2}$ The Royal Marsden NHS Foundation Trust, Sutton \& London, United Kingdom

${ }^{3}$ Department of Obstetrics and Gynaecology, Prince of Wales Hospital, Hospital Authority, Hong Kong SAR, China

4Department of Obstetrics and Gynaecology, Princess Margaret Hospital, Hospital Authority, Hong Kong SAR, China

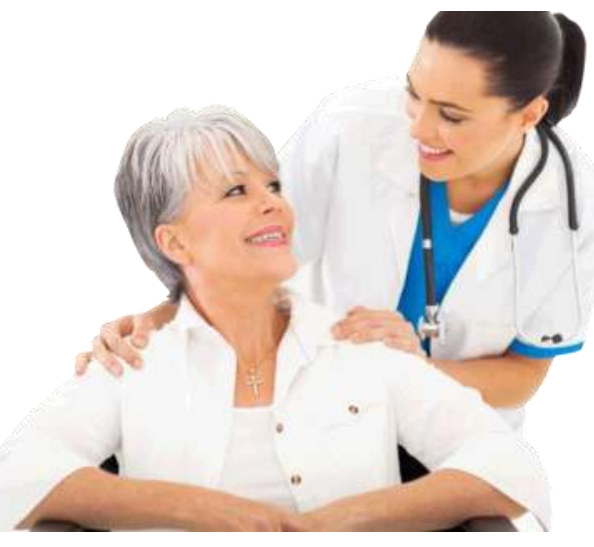

The work described in this study was fully funded by General Research Fund (GRF) from Research Grants Council, the Government of the HKSAR (Project No. 14620316)

\section{Introduction}

* Gynecological cancer (GC) refers to cancers involving the female reproductive tract

* According to the National Cancer Institute (2012), $\mathbf{5 0 \%}$ of GC survivors suffered from long-term sexual dysfunction

* Any adverse changes in sexual functioning may reflect problems in all areas of psychosocial aspect and quality of life (Molassiotis, Chan, Yam, \& Chan, 2000; Stead, Brown, Fallowfield, \& Selby, 2003)

* Information needs of GC patients (Rasmusson \& Thomé, 2008)

* They wanted to get sufficient information about the sexual consequences of GC and treatment from doctors or nurses

* Such knowledge would minimize the risk of any negative effects on a couple's relationship

* In Chinese culture, sexuality is viewed as a taboo topic (Tsa et al., 2011)

* In Hong Kong, both care and interventions in the area of sexuality are still neglected

\section{Aim}

This study aimed to develop a practice model and local protocol to guide and promote the provision of sexuality nursing care to Hong Kong GC patients

\section{Phase}

\section{Methods}

\section{- Literature review and qualitative interviews}

- To explore and elicit perceptions of sexual functioning and ideas about good nursing practice in sexuality care

- $30 \mathrm{GC}$ patients

- 30 spouses/partners

- 20 Registered Nurses and Physicians working in the gynaecological oncology unit

\section{Phase I}

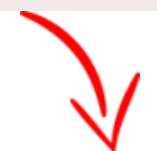

Same participants rated the the level of similarities and perceived importance as to how the statements generated in Phase I affected

- Acceptability

- Appropriateness

- Feasibility of providing sexuality care

- A concept map illustrating the relationships and clustering between the statements

\section{Phase II}

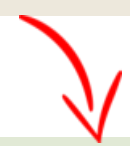

- The concept map was used to inform the development of a practice model and local protocol

To guide nurses and promote the provision of timely, effective and economical nursing care in the area of sexuality

The newly-developed protocol was returned to 17 nurse and physician participants for further validation

References.

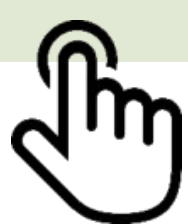

\section{Protocol For Sexuality Care For GC Patients}

\section{Standard Statement}

All GC patients' needs for sexuality care are assessed, and appropriate intervention and support are provided to address these sexual healthcare needs.

\section{Expected Outcomes}

1. GC patients will receive sexuality care in clinical setting.

2. Healthcare professionals will proactively deliver individualised sexuality care to GC patients.

Structure Standard

The gynae-oncology care team includes:

$>$ Protocol introducer\#

Healthcare professional ${ }^{\#}$

$>$ Organizational support \#

\section{Process Standard}

This process standard was guided by the Extended PLISSIT model (Taylor \& Davis, 2006), a schematic representation of which is shown in Figure 1. Findings from the study were incorporated into each phase of the Extended PLISSIT model to guide sexuality nursing care for Hong Kong GC patients.

1. Permission-giving stage\#

2. Limited Information stage

3. Specific Suggestions stage

4. Intensive Therapy stage ${ }^{\#}$

5. Reflection and review\#

\section{Outcome Standard}

1. GC receive sexuality care in clinical setting

2. Healthcare professionals proactively deliver sexuality care to GC patients.

\#Details not shown here

Validation result ( $\mathrm{n}=17$ nurse and physician participants)

$\Rightarrow 88.2 \%$ found the protocol acceptable or very acceptable.

$\rightarrow 82.4 \%$ found the protocol appropriate or very appropriate.

$\rightarrow$ Some participants raised concerns about insufficient resources and training. There were also a few suggestions on the topic format, timing of provision and priority setting.

\section{Discussion}

The newly developed protocol could help nurses to render more effective assistance to GC patients, their spouses/partners in adapting to the changes in sexuality after diagnosis and treatment.

- From the perspective of healthcare services, the findings of this study could inform women's health development, as well as serving as a reference point for sexuality care in other countries with Chinese population.

Further study should explore the feasibility of territory-wide dissemination of this protocol.

Molo

National Cancer Institute (2012). Sexuality and Reproductive Issues. Retrieved from: http://www.cancer.gov/cancertopics/pdq/supportivecare/sexuality/HealthProfessional. Rasmusson E., Thomé B. (2008). Women's wishes and need for knowledge concerning sexuality and relationships in connection with gynaecological cancer disease. Sex Disabil, 26(4), 207-18. Stead M.L., Brown J.M., Fallowfield L., Selby P. (2003). Lack of communication between healthcare professionals and women with ovarian cancer about sexual issues. Br J Cancer, 88(5), 666-71 